silver wire for all the sutures, buried and superficial. In Mr. Bloodgood's voluminous work on hernia already mentioned, ${ }^{5}$ a long series of cases are given in which the canal was narrowed or obliterated by silver wire; in some of them the rectus muscle was dragged down to Poupart's ligament by means of it. The silver wire is supposed by the writer to exert an antiseptic action on the tissues, an action which can only be superfluous if asepsis be obtained. To my mind no region of the body is less suitable for burying silver wire than the groin and its drawbacks were experienced by Mr. C. B. Lockwood, ${ }^{7}$ who tried the method in 15 cases with dismal results. In Bloodgood's cases, whatever was the result as regards recurrence of the hernia, the number of troublesome complications recorded is most remarkable. Induration of the epididymis was even referred to as being usual (p. 428) and orchitis followed by atrophy of the testis was frequent. Yet the result in the latter cases was termed "perfect" (p. 480 and elsewhere). Hæmatoma of the scrotum, sloughing of part of Poupart's ligament (p. 427), cedema of the lower limb from obstruction of the femora vein (three cases), and other complications which could be quoted make up a formidable list which should deter surgeons from using these rigid wire sutures in the radical cure of hernia.

The operation on femoral hernia.-I have only succeeded in following up 16 cases for more than two years, 14 women and two men. The average period at which they were last traced was four and a half years after operation, the average age of the patients at the time being 42 years. There were two recurrences-i.e., 12 per cent. In one of these the recurrence took place three years after operation. In the other case the patient was nearly 60 years of age and had chronic bronchitis. She would not have been operated on for radical cure but for the fact that the hernia was strangulated. Although the number of cases is not large it bears out the statement of Mr. Bloodgood ${ }^{8}$ that "the cure of a femoral hernia is not a difficult problem."

I have occasionally turned up a flap of pectineus muscle to close the ring, but in the great majority of cases $I$ have twisted and fixed the neck of the sac high up with kangaroo tendon and then sutured with the same material Poupart's ligament to the deep fascia, using two or three separate buried sutures. One has to be careful to avoid injurious pressure on the femoral vein ; in one case there was evidence of this, the patient's leg swelling from time to time. In one case of strangulated femoral hernia interesting evidence was obtained of the efficiency of the method alluded to. The patient was an old woman who was suffering from bronchitis, from which she died four days after the operation. At the post-mortem examination $I$ found the femoral ring to be well closed ; it resisted firm pressure from above made with the finger.

I have had no personal experience with Roux's method of radical cure-fixation of Poupart's ligament to the pubis by means of a metal staple. Mr. J. Crawford Renton ${ }^{9}$ records 25 cases of its use and speaks highly of it. Roux is said to have operated 136 times in this way with only two recurrences of the hernia. The objections that might be raised-viz., that the staple would be liable to work loose and that the femoral vein might be compressed too much-are presumably without much ground. The method seems especially indicated where the femoral ring is widely patent.

One point in the after-treatment of radical cure may here be alluded to-the length of time requisite to secure a sound scar. In both inguinal and femoral cases the wound is, as a rule, soundly healed at the end of a fortnight or three weeks. Is it necessary to insist on the patient resting for more than this time? Some operators, I believe, require two or three months' abstention from work on the part of thei patients. But with working men this is a very serious consideration and may mean the entire loss of their previous employment. I see no reason for believing that such prolonged rest is required and have therefore never advised it.

In conclusion, with regard to the radical cure of inguinal hernia the following observations may be made: (1) In all but the simplest cases in children it is best to open up the canal and to narrow it by deep sutures; (2) suturing the conjoined tendon to Poupart's ligament behind the cord by a series of interrupted sutures is probably the best method of

6 Bloodgood and Halsted : Johns Hopkins Reports, 1900. 7 Lockwood: Transactions of the Medical Society of London, 1905 p. 168 .

Johns Hopkins Reports, 1900, p. 365.

9 Contributions to Clinical Surgery, p. 140. narrowing the canal; (3) where the conjoined tendon is deficient, in all recurrent cases, and in inguinal hernia in women the canal should be obliterated ; (4) kangaroo tendon is admirably adapted for the suture material, silver wire being probably the worst; (5) recurrence of the hernia in situ may possibly take place at any length of time after the operation, but if two years be adopted as the limit it should not occur in more than from 5 to 8 per cent.; and ( 6 ) the development of a hernia at another site after operation may be expected in a proportion of cases at least as large as that ust given.

Park-crescent, W.

\section{ON THE TREATMENT OF CANCER BY THERAPEUTIC INOCULATIONS OF A BACTERIAL VACCINE,}

CONTROLLED BY MEASUREMENTS OF THE OPSONIC POWER OF THE BLOOD. ${ }^{1}$

BY DR. C. JACOBS,

AGRÉGÉ AT THE FACULTY OF MEDICINE OF BRUSSELS; $\mathrm{AND}$

DR. VICTOR GEETS,

ASSISTAN'T AT THE INSTITUT ST, ANNE; SOMETIME DEMONSTRATOR OF' PHYSIOLOGY IN THE PHYSIOLOGICAL LABORATORY

OF THE UNIVERSITY OF LONDON.

THE researches which we have made in connexion with cancer have convinced us: (1) that we have in cancerous cachexia a specific micro-organism, the micrococcus neoformans of Doyen; (2) that the anti-cancerous sera of Doyen with which we have experimented are wholly useless; and (3) that it is practicable to immunise the human organism by means of a series of inoculations of the micrococcus neoformans vaccine provided that these are properly controlled by examinations of the opsonic power of the blood.

In connexion with the first of these subject matters it will suffice to recall to mind that those bacteriologists who, like Metchnikoff, have sought in the different forms of neoplasia for the micrococcus neoformans by the technique prescribed by Doyen have succeeded in finding the micro-organisms, the cultures obtained in the different laboratories being all identical. We ourselves have cultivated the micro organism from 90 per cent. of the tumours examined. Furthermore we have succeeded in producing localised or generalised neoplasic lesions in 30 per cent. of the cases by inoculating young and vigorous cultures of the micro-organism into mice and white rats. Lastly, as a final argument we shall prove in the course of this paper that the micrococcus neoformans is without doubt the agent of cancerous cachexia.

With regard to the serum of Doyen the clinical results were negative, as our experimental researches made on laboratory animals had led us to expect. This serum was destitute of bactericidal, bacteriolytic, agglutinating, or opsonic power. This serum, had it really possessed the requisite qualities, would have been a precious instrument in the hands of the therapeutist for raising rapidly the defensive power of the cachectic individual; it ought to be discarded.

The vaccines, on the contrary, can give results in careful hands but their inoculation should be made under constant control if it is desired to avoid the bad results which we soon found out when using them. In fact, the vaccines of Doyen are unstandardised vaccines. In view of the principles of immunisation as expounded by Wright we grasped one after another the "why" of the variations, of the mortifications or the negative results obtained by the inoculation of doses of vaccine in the rule-of-thumb manner as advocated by Doyen.

To avoid these accidents we propose the employment of vaccine made from cultures of the same age, sterilised at $60^{\circ} \mathrm{C}$., washed freely, and standardised by the enumeration of the bacilli as advocated by Wright. The antibacterial reaction, which is the method of protection of the body, must be closely watched after inoculation. The method which we have employed is based on the plan of defence employed

1 Synopsis of a paper communicated to the Royal Belgian Academy Medicine on Jan. 27th, 1906. 
by the body in its struggle against the micrococcus neoformans. This plan is neither bactericidal nor bacteriolyticit is phagocytic. This phagocytosis takes place owing to the presence of an element capable of combining with the micrococcus neoformans and of preparing it for intracellular digestion. This substance, named "opsonin" by Wright (from opsono, I prepare), can be discovered and measured in a drop of blood by means of the technique of Leishman, modified by Wright and Douglas. The blood of all our cancer patients was found below normal with respect to its opsonic action upon the micrococcus neoformans; and the opsonic index undergoes variations in strength after each injection of the micrococcic vaccine. The selection of the dose of vaccine and of the appropriate times for the inoculations was based on the examination of the alterations in the opsonic power of the blood. These variations have been carefully studied for a year in all patients subjected to the vaccine treatment and we wish to lay before you the experience gained by us with regard to the rational treatment of cancerous patients.

The general conclusion which emerges from our study is that in certain cases the vaccine can do much, in others it can do nothing. Just as a tubercle vaccine judiciously administered does not cure all tuberculous cases, so a "cancer vaccine" cannot cure all cancerous cases. This question of success or failure in cases apparently alike depends entirely upon the reserve of vital energy and the capacity for reaction which each patient possesses, and this differs in each case. With regard to the immunising we can express a definite prognosis after two or three injections of the vaccine, the alterations effected in the quantity of the opsonins expressing accurately the strength of the auto-protective reaction. For example, when the opsonic index does not rise after one or two injections the case is irremediably lost, the immunity has fallen to the lowest degree, the defensive powers seem exhausted and cannot be stimulated by anything. Continued injections in such cases can only hasten the death of the patient, since each injection of the vaccine removes a certain amount of the protective substances, and as no phase of manufacture, no positive phase, succeeds to this, we only set up a dangerous cumulation of negative phases, leading the patient to an early death.

Let us now consider what occurs in the most favourable cases after an appropriate dose of vaccine has been inoculated. Let us imagine a cancerous patient the examination of whose blood reveals an opsonic index below normal, say, 0.7 . An injection of the vaccine is now made. A blood examination undertaken several hours later shows a more or less marked negative phase, more or less transient, with the auto-protective power of the blood lowered to 0.5 . Two days later a positive phase succeeds it; an abundant production of protective substances has occurred; the blood possesses defensive powers superior to those met with in the normal individual, and the opsonic index has risen to $1 \cdot 2$. This remains constant for a variable time and then shows a tendency to return to its former level. The time has now come to intervene with a second injection. This done we again see the succession of negative phase, positive phase, plateau of increased protective power. and subsequent descent; with this difference, that the lowest level to which the opsonic power is reduced in the negative phase is 0.8 , while the immunity in the positive phase is raised to $1 \cdot 8$, nearly double the normal strength of the blood. These fluctuations in the protective power of the blood have been described by Wright in the phrase "ebb and flow and reflow and maintained high tide of immunity," and again in the phrase "cumulation in the direction of the positive phase." When the curve of immunisation follows this course the patient is one in whom the cancerous development seems arrested. Locally, the surrounding infiltration decreases, the cancerous nodules are reduced to their actnal size, the tumour seems to fade, and the skin loses its inflamed appearance. In the case of intra-abdominal tumours this improvement is manifested by the fact that the tumours become moveable and by the fact that their limits can now be defined. The general condition is rapidly modified and the most striking fact observed at the beginning of the treatment is that the patient loses his cachectic colour, the pain is diminished, and, in short, the disease seems to be arrested. This is the time when surgical intervention is likely to be successful if the disease is situated in an organ which is removeable. We advise that this interference should, as formerly, be as extensive as possible, for it cannot be doubted that symbiosis with a mass of newly formed-shall we say teratological ?- tissue must go hand in hand with the elaboration:and absorption of products injurious to the organism.

To take away a neoplastic mass is to suppress a constant source of cellular toxins in an organism which is already painfully struggling against an infection which is infiltrating everywhere. It is quite evident that we cannot expect an anti-neoplastic vaccine to cause softening or complete disappearance of large tumours in a few days and even supposing that it were possible to bring about a necrotic change in a large tumour the organism would not be capable of dealing with and eliminating more than a limited quantity of the products of such cellular metabolism. It would succumb to the harmful effects of an excess of this cellular débris. We cannot demand a therapeutic method to perform more than it can reasonably be expected to do. If it is capable only of holding in check a disease otherwise confessedly incurable we ought, therefore, to employ it as early as possible. We have more chance of succes $s$ in our attempts to produce active immunisation if we take the disease at the start, when the patient still bas much undeveloped defensive power which otherwise would be used slowly and uselessly in such an unequal and insidious contest as is waged between the organism and the invading neoplasm.

Unfortunately, our task is the most difficult, because the patients almost always come to us in a more or less advanced cachectic condition; only with comparatively few can we produce the curve of immunisation which would lead to their cure. What should we do in such cases? The examination of the blood will assist us. The lower the opsonic index the more cautiously should we proceed by inoculating the smallest initial dose of vaccine so as to avoid too marked a negative phase. As soon as a positive phase is obtained reinoculation is to be resorted to only when that curve begins to decline. We had, in point of fact, in the days before we instituted the method of control which now guides all our operations, recognised that patients who appeared to improve markedly at the beginning of the treatment presented, when the treatment was pursued, a sudden aggravation of their local and general condition-an aggravation which was in every case definite and incurable.

To present a concrete picture of the events which supervene upon inoculations conducted as we now conduct them we shall sum up observations on a few patients showing the different results observed in cases as they present themselves in practice.

1. Case treated solely with a neofurmans vaccine showing a typical immunisation curve.-A patient, aged 56 yєars, under the care of Dr. Degueldre of Anvers, came to us 12 months ago suffering from inoperable cancer of the rectum with signs of profound cachexia in addition to constant functional troubles. On admission ber opsonic incex was found to be $0 \cdot 7$. The vaccine injections immediately started, safeguarded by inoculations as explained above, gradually raised the protective substances of the blood to $0 \cdot 8,0 \cdot 9$, and after sereral months to $1 \cdot 2,1 \cdot 1$. To-day the patient has no symptom beyond slight constipation. The general condition is excellent; the characteristic complexion has disappeared, giving place to a perfectly normal appearance. Rectal examination reveals an indurated ring situated high up, quite mobile; this is all that remains of the enormous immoveable mass found before the treatment. The present lesion has remained staticnary for more than ten months.

2. 1 case treated with therapeutic inoculations of a neoformans raccine and surgical interferenct; typical immunisation curve and "cure."-The patie $=t$, ag cd 64 years, was suffering from cancer of the body of the uterus with very pronounced cachexja. Six weeks before we saw her she had undergone a palliative opesation, performed by Frofesscr Kufferath. The blood examination reveaîed that the opsonic index was below normal-0.65. Three vaccine injections were administered which brovght up the opsonic index from 0.65 to 0.9 . At the same time the general condition was improved; the uterus became moveable and justified a radical operation which had been considered impossible three weeks previously. Since the operation the opsonic index bas risen above normal and we confine ourselves at present to maintaining the defensive properties of the blcod at this level by injections at long intervals. The 
patient enjoys excellent health ; there is no trace of recurrence; we can hope that she will be eventually immune. The operation took place seven months ago.

3. Typical ourve followed by sudden fall consequent on excessive injections. - The patient, aged 54 years, was suffering from recurrence of cancer of the breast; there were a cancerous growth along the scar, great odema of the right arm, and agonising pain. The opsonic index at the time of admission was 0.78 . Injections given at once raised the phagocytic value above normal-to $1 \cdot 2$. Healthy skin reappeared in certain parts of the cancerous growth, the patient could move the arm and the fingers, and the pain diminished. Daring 2) days two injections kept the index at 1.2. The last scarcely raised it; nevertheless, we repeated it and the last inoculation made the opsonic index rapidly fall below normal-to $0 \cdot 85$. The patient, abandoned defencelessly to the infectious invasion, soon exhibited metastases, intestinal, medullary, and locally the cancer slowly invaded the whole thorax. The opsonic index progressively descended to $0 \cdot 77$, $0 \cdot 7$, and $0 \cdot 6$. The patient died six weeks after the curve of immunisation had commenced its descent.

4. $A$ case of cancer of acute course; neqative curve.-The patient, aged 24 years, underwent in January, 1905, ampu tation of the posterior lip of the cervix for ectropion. Histological examination showed that the tumour was an epithelioma. Radical operation was advised but was refused by the patient. A month later the disease recurred and appeared on what was left of the cervix. In June, 1905, completely worn out by continuous bleeding, the patient consented to undergo hysterectomy. She already presented the characteristic cachectic complexion. Abdominal hysterectomy was performed and at the same time the infected lymphatic glands were removed as far as possible. The blood examination showed the opsonic index to be below normal, $0 \cdot 5$. Cautious injections were started but the opsonic index oscillated between $0.5,0.51$, and 0.48 . The patient gave no stronger reaction. Recurrence "was immediate, pelvic and vaginal. Metastases soon appeared in the liver, the stomach, and the intestine. The general condition became pitiable; the pain was frightful in spite of large doses of morphine. The injections were, however, cautiously continued. The opsonic index rose a little, reaching 0.5 at the same time as the general condition somewhat improved. The morphine was decreased and the pain lessened but the invasion of the cancer followed its prograssive course. Notwithstanding the improvement which we noted and which corresponded accurately with the results of the blood examination the prognosis remained very gloomy and we expected a fatal issue after a brief arrest. In spite of all care the opsonic index remained much below normal and the patient died in an advanced cachectic condition in December, 1905

5. Positive curve; generalised abdominal canoer; profound cachexia. - A patient, aged 34 years, a primipara, under the care of Dr. Moeller, Dr. Capart, and Dr. Cuypers at Neerpelt, had been ill for two and a half years. She had been operated upon for a uterine disease several years ago and in 1904 posterior colpotomy with vaginal drainage had been performed to evacuate a collection in the right side of the pelvis which was believed to be purulent and of appendical origin. The pelvic collection proved to be ascites set up by a carcinomatous lesion of the tubes. Then followed progressive extension of the tumour masses to the entire abdomen and at the same time rapid cachexia. In February, 1905, abdominal section was performed, allowing examination of the extent of the lesions; all the abdominal organs were bathed in an abundant hæmorrhagic ascitic fluid and were themselves affected; the peritoneal cavity was crowded with tumours of all sizes, tumours which, on histological examination, were shown to be small-celled myxosarcoma originating from the uterine tubes. The operation was incomplete, the precarious state of the patient rendering a prolongation of it impossible. There was immediate return of the new growth, and the cachexia increased; the bladder, the stomach, and the intestine were gradually invaded. The situation was regardeo as / st, all nourishment having become impossible. At that time the opsonic in lex was below normal. A vaccine injection was given, followed very soon by an extraordinary increase of the phagocytic power. With incredible rapidity we saw the grave symptoms improve, the vomiting ceased, the intestine resumed its functions, and the urine became clear Still taking as our guide the determinations of the opsonic index we continued the vaccine injections. One and a half months after the beginning of the treatment the patient got up for several hours daily. She has now gained more than 13 kilogrammes in weight and has resumed her work. Locally the tumour masses have disappeared except at the bottom of the pelvis, where, however, the uterus is becoming gradually moveable. All ascites has disappeared and menstruation has become regular. This extraordinary result has been verified by the medical men in attendance.

We have selected from the 46 cases which our statistics now comprise those which appear to us most instructive for the clinical demonstration of our researches. We are far from speaking at present of an absolute cure; time alone can verify the "cures" which we report to-day. In all the cases which we have treated by this method we have noticed real and definite improvement. Where the body was worn out by the cancerous intoxication the improvement was only transient, but it has persisted after several months in the patients where the treatment (with or without operation) has been persisted in; these cases remain in a state of health which contrasts in a most striking manner with their former condition of cachexia. Our statistics of 46 cases are grouped as follows:-

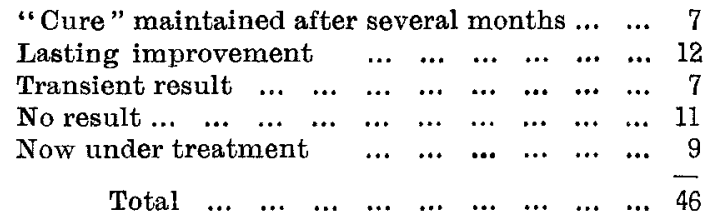

In conclusion, we desire to express our indebtedness to Professor A. E. Wright and to his fellow-worker, Captain S. R. Douglas, I.M.S., to the former for generous scientific hospitality and learned counsels, to the latter for admirable demonstrations of technique accorded to one of us in the pathological laboratory of St. Mary's Hospital preliminary to the inception of this research.

Brussels.

\section{Clinital atotes:}

\section{MEDICAL, SURGICAL, OBSTETRICAL, AND THERAPEUTICAL.}

\section{NOTE ON A CASE OF FACIAL PARALYSIS SUCCESS.} FULLY TREATED BY OPERATION IN AN INFANT.

\author{
By arthur Court, M.B., B.C. Cantab.
}

THE patient, a child, aged 17 months, was admitted to the Marsden Moor isolation hospital on Dec. 15th, 1905. He had already been ill seven days with an attack of scarlatina anginosa. He was partially collapsed, with a feeble pulse at the wrist too rapid to count. There was considerable inflammation of the fauces, which were coated with tenacious mucus. The breath was foul and there were an infective rhinitis and profuse discharge of offensive pus from both ears. From the 15 th to the 27 th his temperature ranged between $101^{\circ}$ and $103^{\circ} \mathrm{F}$. and his general condition remained critical. He was put on a stimulating diet and his throat was sprayed every three hours with an antiseptic lotion. The ears were irrigated with warm boric and carbolic solutions alternately. On the $27 \mathrm{th}$ slight right facial paralysis was first detected; by the 29 th it was complete. There was post-aural tenderness and the patient was fretful, vomiting several times. On inspection there were found to be a large perforation in the drum and sagging of the postero-superior meatal wall. On Jan. 2nd the temperature returned to normal and by the 7 th the nose and throat were nearly clean, but the child was in a very feeble and exhausted condition. The ears were still being treated with the lotions and with instillations of peroxide of hydrogen twice daily.

It was not until Jan. 26 th that the patient's general health would admit of any operative treatment, but as there was no improvement in the character of the discharge and the mastoid tenderness was still present I proceeded to perform the complete post-aural operation. Dr. A. S. Allum administered the anæsthetic. The antrum was full of offensive pus and when the outer attic wall had been removed a condition of acute caries was found to be present in the tym. panum and attic. All the diseased lining was curetted away, 